\title{
Changing from a two-tiered to a one-tiered trauma team activation protocol: a before-after observational cohort study investigating the clinical impact of undertriage
}

\author{
Kenneth Thorsen ${ }^{1,2,4}\left(\right.$ D $\cdot$ Jon Kristian Narvestad ${ }^{1,2} \cdot$ Kjell Egil Tjosevik $^{3} \cdot$ Johannes Wiik Larsen $^{2} \cdot$ Kjetil Søreide $^{2,4}$
}

Received: 15 January 2021 / Accepted: 4 May 2021 / Published online: 22 May 2021

(c) The Author(s) 2021

\begin{abstract}
Background The aim of this study was to compare the effect of the change in TTA protocol from a two-tier to one-tier, with focus on undertriage and mortality.

Material and methods A before-after observational cohort study based on data extracted from the Stavanger University Hospital Trauma registry in the transition period from two-tier to a one-tier TTA protocol over two consecutive 1-year periods (2017-2018). Comparative analysis was done between the two time-periods for descriptive characteristics and outcomes. The main outcomes of interest were undertriage and mortality.

Results During the study period 1234 patients were included in the registry, of which 721 (58\%) were in the two-tier and $513(42 \%)$ in the one-tier group. About one in five patients (224/1234) were severely injured (ISS > 15).

Median age was 39 in the two-tier period and 43 years in the one-tier period $(p=0.229)$. Median ISS was 5 for the two-tier period vs 9 , in the one-tier period $(p=0.001)$. The undertriage of severely injured patients in the two-tier period was $18 / 122$ (15\%), compared to $31 / 102$ (30\%) of patients in the one-tier period ( $\mathrm{OR}=2.5 ; 95 \% \mathrm{CI} 1.8-4.52)$. Overall mortality increased significantly between the two TTA protocols, from 2.5 to $4.7 \%(p=0.033)$, OR $0.51(0.28-0.96)$

Conclusion A protocol change from two-tiered TTA to one-tiered TTA increased the undertriage in our trauma system. A two-tiered TTA may be beneficial for better patient care.
\end{abstract}

Keywords Trauma $\cdot$ Trauma team $\cdot$ Trauma team activation protocol $\cdot$ Mortality $\cdot$ Injury severity

\section{Introduction}

Trauma is a leading cause of death and disability for people $<45$ years of age worldwide, including the Nordic countries [1-3]. To minimise the consequences of trauma, optimal care in all stages of the trauma management are essential [4]. Protocols have been put in place to ensure trauma team

Kenneth Thorsen

kenneththors@gmail.com

1 Section for Traumatology; Surgical Clinic, Stavanger University Hospital, Stavanger, Norway

2 Department of Gastrointestinal Surgery, Stavanger University Hospital, PO Box 8100, 4068 Stavanger, Norway

3 Department of Emergency Medicine, Stavanger University Hospital, Stavanger, Norway

4 Department of Clinical Medicine, University of Bergen, Bergen, Norway activation (TTA) for patients who are severely injured or believed to be at risk of serious injury. The jeopardy of any protocol and its criteria is the risk of either over- or under-triage [5]. When a trauma patient with a major injury, defined as ISS $>15$, is not met by a trauma team, the patient is undertriaged [5]. Undertriage may be the result of too stringent criteria for TTA, failure to recognise the criteria in the prehospital field or due to an inappropriate staffed team. Such undertriage may be associated with longer time to diagnosis, longer time to lifesaving interventions and higher risk of death $[6,7]$. To avoid undertriage, criteria are set to allow for a certain degree of overtriage. An undertriage of $<5 \%$ and an overtriage of $25-50 \%$ has been proposed as acceptable by the American College of Surgeons [8].

However, several studies from Norway has described considerable higher undertriage than the accepted $5 \%[5,9,10]$ and also considerable higher overtriage than accepted, of about $80 \%$ [10]. 
A previous report found a favourable effect of revising TTA to a two-tiered team protocol, with a regular full 13-member team activation for all major trauma and a reduced team given specific criteria for TTA [5]. The twotier protocol led to a significant reduction in undertriage from 28.4 to $19.1 \%$ after system revision. However, with mandated implementation of a national trauma plan, the institutional TTA protocol was returned to a one-tier TTA, without good data to support this decision. Hence, the current study was designed to evaluate the effect of going back to a one-tier protocol from the previously reported two-tier TTA.

The aim of this study was to compare two time periods before and after TTA protocol change with focus on the impact on undertriage, characteristics of undertriaged patients and mortality.

\section{Material and methods}

\section{Ethics}

This study was accepted as a quality improvement project by the personal data officer in Stavanger University Hospital. The SUH trauma registry also has approval from the personal data officer as a quality registry.

\section{Study design}

A retrospective, consecutive, observational cohort based on a before-and-after evaluation of two time periods, the "before-period" with a two-tier system compared the "after-period" following system revision back to a one-tier TTA. The study is performed and reported according to the STROBE guidelines where applicable [11].

\section{Study population}

All data were extracted from the Stavanger University Trauma registry from 2017 to 2018.

For the undertriaged patients the electronic patient journals were also investigated with regards to identifying potential relative trauma criteria present in the patient chart.

The Stavanger region serves a population of about 370.000 people and receives injured patients from about 500.000 inhabitants in a wider catchment area. Annually, about 550 patients are admitted to SUH for suspected or potentially severe trauma and about one in five of the admitted patients have an ISS $>15$. Since January 1, 2004 a formal trauma registry has been in place, including all patients admitted with a trauma alarm and patients with an ISS $\geq 9$ not receiving a trauma alarm from pre-set criteria [12].
Patients admitted to the emergency department without trauma team activation but who are found to have an Injury Severity Score (ISS) $>9$ on diagnostic screening or, have a penetrating injury to the head/neck/torso proximal to the elbow or knee, head injury with Abbreviated Injury Scale (AIS) $\geq 3$ or $\geq 2$ proximal long bone fractures are registered in the trauma registry by the trauma registrars. Patients with mild head injuries or isolated femoral neck fractures are not routinely included in the registry.

Patients with a trauma alarm found to have only a medical issue were excluded. Patients found dead at scene were also excluded.

\section{Definition of time periods}

The before period was set from January 1 st to December 31st, 2017. In this period a two-tier TTA protocol was used, as previously described [5]. A full TTA (13 team members) was initiated after prespecified criteria. A reduced TTA (surgeon on-call + ED staff) was initiated according to a limited set of criterias, described previously [5].

The after period was from January 1st to December 31st, 2018. In this period a one-tier protocol with only a full TTA, was applied.

\section{Definitions}

Severe injuries were defined as an injury severity score (ISS) $>15$ [13]. The Association for the Advancement of Automotive Medicine-Abbreviated Injury Scale 1990 revision, update 98 (AIS 98) [14] was used, since this version derived the ISS $>15$ threshold for defining major trauma.

\section{Over- and under-triage}

Overtriage is defined as any TTA for trauma patients with an ISS $\leq 15$. Undertriage is defined as the lack of TTA for a trauma patient with a severe injury defined by an ISS $>15$ [8].

\section{Statistics}

Statistical analysis was performed with The Statistical Package for Social Sciences, SPSS ${ }^{\circ}$ version 26 for Mac (IBM, Armonk, New York, USA).

Descriptive analysis was performed with Chi-squared test or Fisher's exact, where appropriate for categorical variables and Kruskal-Wallis test or Mann-Whitney $U$ test for continuous variables.

All tests are two-sided and a $p$ value of $<0.05$ deemed statistically significant. 


\section{Results}

The patient inclusion criteria are shown in the flow chart (Fig. 1), resulting in 1234 patients in total, divided in 721 patients in the two-tier period versus 513 patients in the onetier period. Patient characteristics comparing the one-tier versus two-tier period are shown in Table 1 and composition of the trauma team in Table 2.

Median ISS was 5 for the two-tier period vs 9 , in the onetier period $(p=0.001)$.

A total of 224 patients had an ISS $>15$ and 49/224 (21.9\%) were undertriaged.
Of the patients with ISS > 15, 18/122 (14.8\%) of patients were undertriaged in the two-tier period compared to $31 / 102$ $(30.4 \%)$ in the one tier period $(p=0.002$, OR 2.5 (95\% CI 1.4-4.5).

The under- and overtriage per cohort, as well as the associated mortality is given in Table 3.

All the undertriaged patients had a blunt trauma mechanism and no difference in undertriage between gender was encountered $(p=0.899)$.

Undertriaged patients were further characterised by a median age of 66 , while those who were not undertriaged had a median age of $38(p<0.001)$.

Fig. 1 Patient flow chart

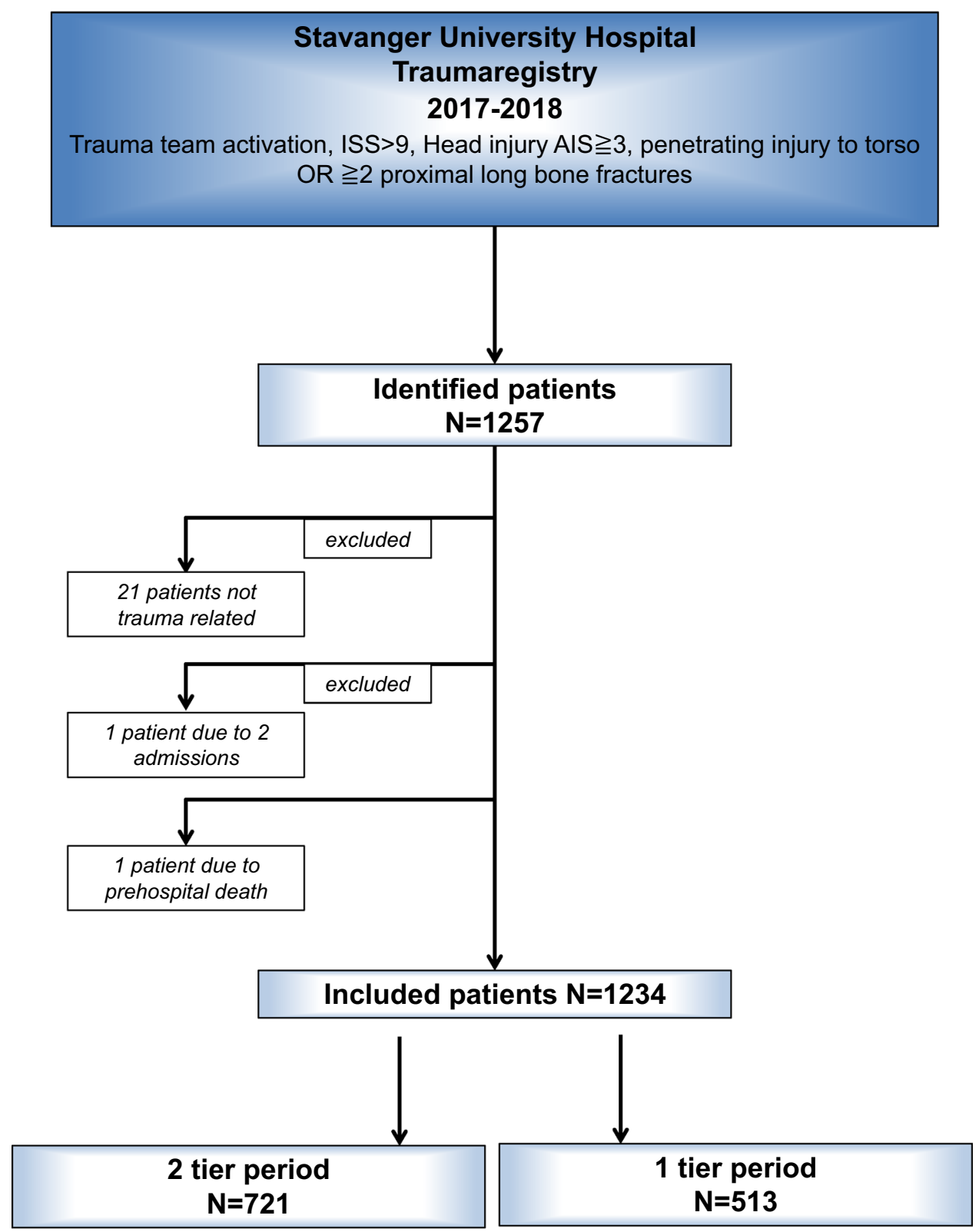


Table 1 Comparison of patients in the 2-tier vs 1-tier TTA cohorts

\begin{tabular}{lccccc}
\hline Category & Total & 2-tier TTA & 1-tier TTA & $P$ & OR (95\% CI) \\
\hline Period, $n(\%)$ & $1234(100 \%)$ & $721(58 \%)$ & $513(38 \%)$ & $<0.001$ & \\
Gender & & & & & \\
Female, $n(\%)$ & $413(33.5 \%)$ & $233(32.2 \%)$ & $180(35.2 \%)$ & & \\
Male, $n(\%)$ & $821(66.5 \%)$ & $488(67.8 \%)$ & $333(64.8 \%)$ & 0.272 & $0.9(0.7-1.1)$ \\
Age (years) & & & & \\
Median, range & $40(0-106)$ & $39(0-106)$ & $43(0-99)$ & 0.229 & \\
Elderly ( $\geq 60$ years), $n(\%)$ & 315 & 170 & 145 & 0.068 & $1.3(1.0-1.6)$ \\
Injury severity & $5(1-75)$ & $5(1-75)$ & $9(1-75)$ & $<0.001$ & \\
ISS, median (range) & $224(18.2 \%)$ & $122(16.9 \%)$ & $102(19.9 \%)$ & 0.183 & $1.2(0.9-1.6)$ \\
Severe injury (ISS $>15), n(\%)$ & $6(1-75)$ & $6(1-75)$ & $9(1-75)$ & 0.001 & \\
NISS median (range) & $314(25.4 \%)$ & $170(23.6 \%)$ & $144(28 \%)$ & 0.074 & $1.3(1.0-1.6)$ \\
Severe injury (NISS $>15), n(\%)$ & & & & & \\
Trauma mechanism & $1198(97 \%)$ & $689(95.6 \%)$ & $489(95.3 \%)$ & 0.642 & $1.1(0.7-1.9)$ \\
Blunt & $62(5 \%)$ & $34(4.7 \%)$ & $28(5.4 \%)$ & & $0.9(0.5-1.4)$ \\
Penetrating & & & & & \\
Mechanism of injury & $544(44 \%)$ & $331(46 \%)$ & $213(41 \%)$ & 0.121 & $1.2(1.0-1.5)$ \\
Traffic, $n(\%)$ & $579(47 \%)$ & $366(51 \%)$ & $213(42 \%)$ & 0.001 & $1.5(1.2-1.8)$ \\
Fall, $n(\%)$ & $64(5.2 \%)$ & $35(5 \%)$ & $29(6 \%)$ & 0.809 & $0.9(0.5-1.4)$ \\
Violence, $n(\%)$ & & &
\end{tabular}

Table 2 Trauma team composition

\begin{tabular}{ll}
\hline Full team & Reduced team \\
\hline Team leader surgeon & Team leader surgeon \\
Orthopaedic surgeon & Orthopaedic surgeon \\
Anaesthetist & Two ED nurses \\
Nurse anaesthetist & \\
Radiologist & \\
Two radiographers & \\
Three ED nurses & \\
Theatre nurse & \\
Laboratory technician & \\
Orderly &
\end{tabular}

When comparing those undertriaged with patients not undertriaged, the patients $>65$ years of age were more likely to be undertriaged $(p<0.001)$, OR 4.5 (95\% CI 2.5-8.0) constituting 25/49 (51\%) of the undertriaged patients, while the age group $>65$ years only constituted $249 / 1234$ (20\%) of the total trauma population. Univariate analyses of associations with undertriage is shown in Table 4.

Most of the undertriaged patients had a relative criteria present. Key elements of every undertriaged patient is shown in Table 5.

It was more likely for patients with fall as trauma mechanism compared to patients without fall as traumamechanism to be undertriaged ( $p=0.001$ ), OR 0.31 (95\% CI 0.16-0.59).

When comparing undertriaged patients with other trauma mechanism than transport injury, they were more likely to be undertriaged than patients with transport injury as trauma mechanism $(<0.001)$, OR 4.26 (95\% CI 1.98-9.17).

Patients that were undertriaged were more likely to succumb within 30 days, than patients who were not undertriaged ( $p<0.001$, OR 0.16 (95\% CI 0.07-0.36).

The criteria for trauma team activation is shown in Table 6 for the before period and in Table 7 for the after period.

There seemed to be somewhat of a cluster of undertriage during winter, with the four winter months:
Table 3 Comparison of outcomes between the two-tier vs one-tier TTA cohorts

\begin{tabular}{lccccc}
\hline Category & \multicolumn{1}{l}{ Total } & Two-tier TTA & One-tier TTA & $p$ & OR (95\% CI) \\
\hline Patients, $n(\%)$ & $1234(100 \%)$ & $721(58 \%)$ & $513(38 \%)$ & & \\
Undertriage & $49 / 224(21.9 \%)$ & $18 / 122(14.8 \%)$ & $31 / 102(30.4 \%)$ & 0.002 & $2.5(1.4-4.5)$ \\
Overtriage & $928 / 1101(84.3 \%)$ & $562 / 665(84.5 \%)$ & $366 / 436(84.0 \%)$ & 0.008 & $0.7(0.5-0.9)$ \\
Correct triage & $257 / 1234(20.8 \%)$ & $141 / 721(19.6 \%)$ & $116 / 513(22.6 \%)$ & 0.98 & $1.0(0.7-1.4)$ \\
Mortality, $n(\%)$ & $42(3.4 \%)$ & $18(2.5 \%)$ & $24(4.7 \%)$ & 0.033 & $0.5(0.3-1.0)$ \\
\hline
\end{tabular}


Table 4 Univariate analyses of undertriaged patients

\begin{tabular}{|c|c|c|c|c|}
\hline & $\begin{array}{l}\text { Undertriaged } \\
\text { patients }(N=49)\end{array}$ & $\begin{array}{l}\text { Not undertriaged } \\
\text { patients }(N=1185)\end{array}$ & $p$ value & OR $(95 \% \mathrm{CI})$ \\
\hline Median age (IQR) & $66(52-83)$ & $38(22-58)$ & $<0.001$ & \\
\hline Median GCS(IQR) & $15(14-15)$ & $15(14-15)$ & 0.747 & \\
\hline \multicolumn{5}{|l|}{ Sex } \\
\hline Male & $33(67 \%)$ & $787(66 \%)$ & & \\
\hline Female & $16(33 \%)$ & $397(34 \%)$ & 0.899 & $1.04(0.57-1.91)$ \\
\hline \multicolumn{5}{|l|}{ Age } \\
\hline$\leq 65$ years & $24 / 983(2.4 \%)$ & 959/983 (97.6\%) & & \\
\hline$>65$ years & $25 / 249(10 \%)$ & $224 / 249(90.0 \%)$ & $<0.001$ & $4.5(2.5-8.0)$ \\
\hline \multicolumn{5}{|l|}{ Transport injury } \\
\hline Yes & $8(16.3 \%)$ & $537(45.3 \%)$ & & \\
\hline No & $41(83.7 \%)$ & $646(54.7 \%)$ & $<0.001$ & $4.26(1.98-9.17)$ \\
\hline \multicolumn{5}{|l|}{ Fall injury } \\
\hline Yes & $36(73.5 \%)$ & $542(45.7 \%)$ & & \\
\hline No & $13(26.5 \%)$ & $636(54.3 \%)$ & 0.001 & $0.31(0-1-0.59)$ \\
\hline Blunt & $49 / 1178(4.2 \%)$ & $1128 / 1178(95.8 \%)$ & & \\
\hline Penetrating & $0 / 56(0 \%)$ & $56 / 56(100 \%)$ & 0.103 & $0.96(0.95-0.97)$ \\
\hline 30-day mortality & $8(16.3 \%)$ & $34(2.9 \%)$ & $<0.001$ & $6.2(2.7-14.1)$ \\
\hline 2-tier period & $18 / 721(2.5 \%)$ & $703 / 721(97.5 \%)$ & & \\
\hline 1-tier period & $31 / 513(6.0 \%)$ & $482 / 513(94 \%)$ & 0.002 & $2.5(1.4-4.5)$ \\
\hline
\end{tabular}

December-March represented 27/50 (54\%) of the undertriaged patients.

After changing from two-tier to one-tier approach the annual admittance rate of trauma patients decreased significantly. The total number of patients with ISS $>15$ was also lower, but not significantly. Median ISS in the two-tier period was 13 for patients with no TTA (Neither full nor reduced) versus five for those with a TTA $(p<0.001)$.

In the one-tier period patients with no TTA had a median ISS of 14 versus five for those with TTA $(p<0.001)$.

Including only patients with an ISS $>15$, the mortality was $13.7 \%(14 / 102)$ in the one-tier period versus $10.7 \%$ $(13 / 122)$ in the two-tier period $(p=0.482)$.

Including only patients with an ISS $\leq 15$, the mortality was $2.8 \%(11 / 393)$ in the one-tier period versus $0.08 \%$ $(5 / 599)$ in the two-tier period $(p=0.019)$.

\section{Discussion}

In the current study, a change in protocol from two-tiered TTA to one-tiered TTA caused a significant increase in undertriage from 15 to $30 \%$, with an odds ratio of 2.5 for undertriage in the after period. The overall mortality increased, but this has to be considered in the light of substantially lower volume of trauma patients admitted in the one-tier period. In addition, due to the change in protocol and lower admittance rate, the patients in the after period had a statistically significant higher median ISS. However, the mortality for the group with ISS $>15$ also increased, but not statistically significant. As such we believe the increase in mortality to be explained first and foremost by the lower denominator in the after period.

The undertriaged patients were dominated by patients $>65$ years of age, with fall as trauma mechanism and head injury as the dominating injury.

The patients that did not receive a TTA had a significantly higher ISS than patients who did receive a TTA. This can be seen as a paradox, yet is, at least to an extent, a function of the current TTA criteria and method of identifying undertriaged patients retrospectively. Further, the patients who did not receive any TTA were significantly older than patients who did receive a TTA. Patients with fall as trauma mechanism were more likely not to receive a TTA than patients with other mechanisms than fall during both periods.

Several other studies have found similar results, with older patients with low-energy falls being undertriaged [5, $9,15]$.There may be several reasons for this. The undertriaged patients in both the before and after period were dominated by geriatric patients with low-energy falls and head injuries as the dominating injury. Most of the undertriaged patients were found to have a relative criterion that would yield a reduced team in the before period, while this did not yield a TTA in the after period, but stated "a lower threshold for TTA present if any of the criteria present". Hence, the lack of a reduced team seems to have given the prehospital personnel a higher threshold for trauma alarm set-off, understanding the consequences overtriage with a full trauma 
Table 5 Undertriaged patients at Stavanger University hospital in the (a) before period (2017), (b) after period (2018)

\begin{tabular}{llllll}
\hline Patient Age sex & Injury mechanism & $\begin{array}{l}\text { Abso- } \\
\text { lute } \\
\text { criteria }\end{array}$ & Relative criteria ISS Dominating injury & $\begin{array}{l}30 \text {-day } \\
\text { mortal- } \\
\text { ity }\end{array}$ \\
\hline
\end{tabular}

\begin{tabular}{|c|c|c|c|c|c|c|c|c|}
\hline \multicolumn{9}{|l|}{ (a) } \\
\hline 1 & 81 & Female & Low-energy fall & No & Yes & 29 & Subdural hematoma & Yes \\
\hline 2 & 21 & Male & Fall from skateboard & No & Yes & 17 & Open tibial fracture & No \\
\hline 3 & 38 & Male & Low-energy fall & No & No & 17 & Epidural/subdural hematoma & No \\
\hline 4 & 52 & Male & Low-energy fall & No & Yes & 29 & Subdural hematoma & No \\
\hline 5 & 65 & Female & Fall & No & Yes & 41 & Liverlaceration/subarachnoidal bleeding & No \\
\hline 6 & 62 & Male & Bicycle & No & Yes & 18 & Subarachnoidal bleeding & No \\
\hline 7 & 92 & Male & Low-energy fall & No & Yes & 17 & Subdural hematoma & No \\
\hline 8 & 69 & Male & Fall & No & Yes & 16 & Subarachnoidal/subdural bleeding & No \\
\hline 9 & 90 & Female & Fall in stairs & Yes & Yes & 26 & Subdural hematoma/costa fractures & Yes \\
\hline 10 & 91 & Male & Low-energy fall & No & Yes & 16 & Hemothorax/multiple costa fractures & No \\
\hline 11 & 77 & Male & Low-energy fall & Yes & Yes & 24 & $\begin{array}{l}\text { Subdural hematoma/subarachnoidal bleeding/skull } \\
\text { fracture }\end{array}$ & No \\
\hline 12 & 89 & Male & Fall in stairs & No & Yes & 17 & Subdural bleeding & No \\
\hline 13 & 61 & Male & Low-energy fall & No & Yes & 17 & Multiple costa fractures & No \\
\hline 14 & 51 & Male & Bicycle & No & No & 17 & Subdural hematoma, $\mathrm{C} 4$ fracture & No \\
\hline 15 & 67 & Male & Low-energy fall & No & Yes & 17 & Subdural hematoma & Yes \\
\hline 16 & 60 & Male & Low-energy fall & Yes & Yes & 25 & Bilateral subdural hematoma & No \\
\hline 17 & 57 & Male & Motorcycle & No & No & 16 & Large pneumothorax, costafractures & No \\
\hline 18 & 63 & Female & Low-energy fall & No & Yes & 17 & Subdural hematoma & No \\
\hline \multicolumn{9}{|l|}{ (b) } \\
\hline 1 & 80 & Female & Low-energy fall & No & Yes & 24 & Subdural hematoma & No \\
\hline 2 & 76 & Female & Low-energy fall & No & Yes & 17 & Intracerebral bleeding & No \\
\hline 3 & 98 & Female & Low-energy fall & No & Yes & 21 & Subdural hematoma & No \\
\hline 4 & 3 & Female & Hit by heavy object & No & Yes & 16 & Skull fracture, cerebellar bleeding & No \\
\hline 5 & 92 & Male & Low-energy fall & No & Yes & 16 & Subdural hematoma & No \\
\hline 6 & 13 & Female & Fall from horse & No & Yes & 17 & Liver laceration grade $4-5$ & No \\
\hline 7 & 7 & Male & Fall & No & Yes & 16 & Spleen injury grade 4 & No \\
\hline 8 & 93 & Female & Low-energy fall & No & Yes & 17 & Subarachnoidal/subdural bleeding & Yes \\
\hline 9 & 44 & Male & Fall & No & No & 16 & Subdural hematoma/skull fracture & No \\
\hline 10 & 50 & Male & MVA & No & Yes & 19 & Multiple costa fractures & No \\
\hline 11 & 59 & Male & Hit by heavy object & No & Yes & 17 & Subarachnoidal bleeding & No \\
\hline 12 & 80 & Male & Low-energy fall & No & Yes & 10 & Small subdural hematoma & No \\
\hline 13 & 6 & Female & Fall & No & Yes & 18 & Spleen injury grade 3 /pneumothorax & No \\
\hline 14 & 48 & Male & Low-energy fall & No & No & 17 & Subarachnoidal/subdural bleeding & No \\
\hline 15 & 48 & Male & Bicyle & No & No & 17 & Subarachnoidal/subdural bleeding & No \\
\hline 16 & 83 & Female & Low-energy fall & No & Yes & 26 & Subdural hematoma & Yes \\
\hline 17 & 66 & Male & Fall & Yes & Yes & 16 & Multiple costa fractures & No \\
\hline 18 & 38 & Female & Bicycle & No & No & 17 & Skull fracture & No \\
\hline 19 & 72 & Male & Low-energy fall & No & Yes & 17 & Subarachnoidal/subdural bleeding & No \\
\hline 20 & 60 & Male & Bicycle & No & Yes & 21 & Subdural hematoma & No \\
\hline 21 & 85 & Male & Low-energy fall & No & Yes & 20 & Multiple costa fractures & No \\
\hline 22 & 82 & Male & Low-energy fall & No & Yes & 17 & Subarachnoidal/subdural bleeding & No \\
\hline 23 & 86 & Male & Low-energy fall & No & Yes & 17 & Subdural bleeding & No \\
\hline 24 & 25 & Male & Sporting injury & Yes & No & 25 & Aortic laceration & Yes \\
\hline 25 & 59 & Male & Low-energy fall & No & Yes & 17 & Subarachnoidal/subdural bleeding & No \\
\hline 26 & 60 & Male & Low-energy fall & No & Yes & 16 & Multiple costa fractures/pneumothorax & No \\
\hline 27 & 72 & Male & Low-energy fall & No & Yes & 21 & Subdural hematoma & No \\
\hline
\end{tabular}


Table 5 (continued)

\begin{tabular}{lllllllll}
\hline Patient & Age & sex & Injury mechanism & $\begin{array}{l}\text { Abso- } \\
\text { lute } \\
\text { criteria }\end{array}$ & Relative criteria & ISS & Dominating injury & $\begin{array}{l}\text { 30-day } \\
\text { mortal- } \\
\text { ity }\end{array}$ \\
\hline 28 & 80 & Female & Low-energy fall & No & Yes & 24 & Neck injury/spinal cord & No \\
29 & 85 & Female & Low-energy fall & No & Yes & 25 & Subdural hematoma & Yes \\
30 & 79 & Female & Fall in stairs & No & Yes & 24 & C7 fracture, medulla contusion, facial fracture & Yes \\
31 & 59 & Male & Fall in stairs & Yes & Yes & 21 & Subdural hematoma/skull fracture/costa fractures & No \\
\hline
\end{tabular}

Table 6 Trauma team criteria in the before period

\begin{tabular}{|c|c|}
\hline Full trauma team & Reduced trauma team \\
\hline $\mathrm{RTS} \leq 11$ & Age $<60$ years \\
\hline GCS $<14$ & Age $<6$ years \\
\hline Respiration rate $<9 /$ min & Severe comorbidity (COPD, heart failure etc.) \\
\hline Respiration rate $>25 / \mathrm{min}$ & Pregnancy \\
\hline Spo $2<90 \%$ & Increased risk of bleeding (anticoagulant drugs, coagulopathy) \\
\hline \multicolumn{2}{|l|}{ Intubated/attempted intubation } \\
\hline Obvious massive haemorrhage & Co-passenger killed \\
\hline \multirow[t]{2}{*}{ Systolic blood pressure $<90 \mathrm{mmHg}$} & Entrapped person \\
\hline & Person ejected from vehicle/motorcycle \\
\hline Facial injury with risk for airway obstruction & Pedestrian, cyclist run down at $>30 \mathrm{~km} / \mathrm{h}$ or thrown up in the air \\
\hline Flail chest & Collision speed $>50 \mathrm{~km} / \mathrm{h}$ \\
\hline Suspected pneumothorax & Deformed vehicle compartment \\
\hline Stab or gunshot wound proximal to knee or elbow & Airbag set off \\
\hline Suspected pelvic fracture & Vehicle roll-over \\
\hline \multicolumn{2}{|l|}{ Crushed, mangled or amputated extremity } \\
\hline Two or more long bone fractures & Fall > 5 m (adults) \\
\hline Open fracture with ongoing haemorrhage & Fall > $3 \mathrm{~m}$ (children) \\
\hline Open skull fracture or impression fracture & Interhospital transfer and $<24 \mathrm{~h}$ since time of injury \\
\hline \multicolumn{2}{|l|}{ Suspected spinal cord injury } \\
\hline \multicolumn{2}{|l|}{ Burn injury ( $\geq$ grade II) $>15 \%$ total body surface area } \\
\hline \multicolumn{2}{|l|}{ Accident with several severely injured (suspected or confirmed) patients } \\
\hline \multicolumn{2}{|l|}{ Upgrade to full trauma team } \\
\hline \multicolumn{2}{|l|}{ When two or more criteria for reduced trauma team are fulfilled } \\
\hline When reduced trauma team finds a perceived stable patient to be unstable & \\
\hline
\end{tabular}

team puts on hospital resources. In some of the cases the trauma criteria were not met, even though the patient later is found to have an ISS $>15$, the corresponding physiologic parameters are not always present. And second, there might exist a prehospital bias to low-energy falls that misleads the prehospital team. The relative criteria in the before period yielded a reduced team, while these criterias are specified as "if any of these criteria are present (see Table 7), a lower threshold for TTA is present" in the current national triage criteria. Ironically, earlier attempts to improve triage precision reduced undertriage from 28 to $19 \%$ after implementing a two-tiered TTA [5]. These results question both the one-tier model and the trauma criterias used for the Norwegian trauma population. However, several studies from
Norway have described considerable higher undertriage than the accepted $5 \%[5,9,10,16]$ and also considerable higher overtriage than accepted, of about $80 \%$ [10] using a one tier TTA model. A fresh Dutch study estimated considerable improvements in triage precision after identifying the most optimal triage criterias in their own trauma population [15].

Efforts have been made elsewhere to address patients $>65$ years of age with low--energy falls, with expedited teams specifically addressed for this group. One study found to decrease length of stay, but with no improvement in mortality after implementing an expedited team [17]. If we are to provide a decent and optimal care for older patients with low injury falls, a change in the trauma system may be necessary. It appears that the trauma criterias in use based 
Table 7 National trauma team criteria in the after period

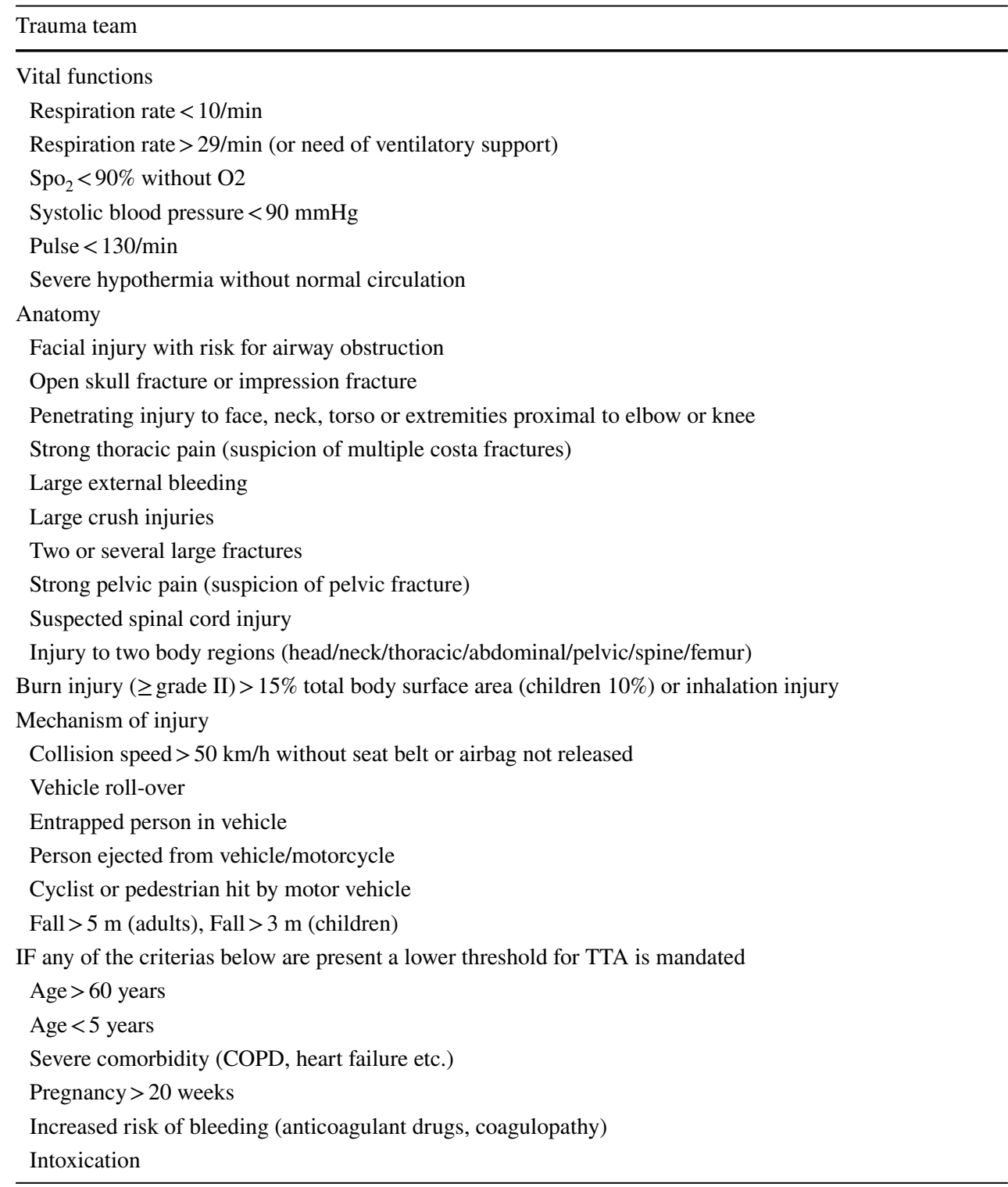

on the field triage criterias does not function optimally for this patient group. As a consequence of the findings in this study, we are introducing a specific geriatric trauma team in SUH, that will seek to address and improve both undertriage and trauma care for the geriatric trauma patients in $\mathrm{SUH}$, specifically for the older patients with low-energy falls and suspected head injury.

Overtriage does not impact the trauma patient negatively per se but may lead to trauma team fatigue by a high rate of "false alerts" or a feeling of "cry wolf" to set alarm criteria. Importantly, other potentially sick patients may suffer in the lack of allocated resources when competing for the same personnel and time. An overtriage of $>80 \%$ as seen in both periods in this study is too high and mandates scrutiny. However, to reduce undertriage one has to accept a certain degree of overtriage, where $25-50 \%$ has previously been described as acceptable by the American college of surgeons.

We question the generic recommendation of one-tiered TTA as recommended in the Norwegian national trauma guidelines and, a change in TTA protocol and/or TTA criteria that is fitted to the need of any specific hospital trauma volume and population seems warranted.

The tendency to a cluster of undertriaged patients admitted in the four winter months was somewhat surprising. We do not have an explanation for this and can only speculate about this finding. It may be just a coincidence since only two years were analysed. But if the finding represents a real cluster, perhaps less sun and potentially vitamin D deficiency can contribute and explain to some degree why there seemed to be a cluster of undertriaged 
patients (geriatric patients with low-energy falls) during the winter months.

Some limitations should be addressed. This is a registry study with its implied strengths and weaknesses. Also, since we chose to directly compare only the year before and after, this could potentially affect the study power yielding potential type II errors due to too low patient volume. It should also be kept in mind that the findings in this single centre study are not necessarily generalizable for other regions and protocols.

\section{Conclusion}

After changing protocol from two-tiered TTA to one-tiered TTA, the undertriage doubled.

Undertriage was associated with falls and age $>65$ years with head injury as dominating injury. An increase in the overall mortality was also observed, but needs to be mirrored by the decrease in the overall trauma admittance rate, yielding a lower denominator in the one-tier period.

Funding Open access funding provided byUniversity of Bergen (incl Haukeland University Hospital).

\section{Declarations}

Conflict of interest None of the authors declare any conflict of interests.

Open Access This article is licensed under a Creative Commons Attribution 4.0 International License, which permits use, sharing, adaptation, distribution and reproduction in any medium or format, as long as you give appropriate credit to the original author(s) and the source, provide a link to the Creative Commons licence, and indicate if changes were made. The images or other third party material in this article are included in the article's Creative Commons licence, unless indicated otherwise in a credit line to the material. If material is not included in the article's Creative Commons licence and your intended use is not permitted by statutory regulation or exceeds the permitted use, you will need to obtain permission directly from the copyright holder. To view a copy of this licence, visit http://creativecommons.org/licenses/by/4.0/.

\section{References}

1. Polinder S, Haagsma JA, Toet H, van Beeck EF. Epidemiological burden of minor, major and fatal trauma in a national injury pyramid. Br J Surg. 2012;99(Suppl 1):114-21.
2. Kristiansen T, Lossius HM, Rehn M, Kristensen P, Gravseth HM, Roislien J, Søreide K. Epidemiology of trauma: a populationbased study of geographical risk factors for injury deaths in the working-age population of Norway. Injury. 2014;45(1):23-30.

3. Søreide K. Epidemiology of major trauma. Br J Surg. 2009;96(7):697-8.

4. Søreide K. Strengthening the trauma chain of survival. Br J Surg. 2012;99(Suppl 1):1-3.

5. Rehn M, Lossius HM, Tjosevik KE, Vetrhus M, Ostebo O, Eken T. Rogaland Trauma System Study Collaborating G: efficacy of a two-tiered trauma team activation protocol in a Norwegian trauma centre. Br J Surg. 2012;99(2):199-208.

6. Dehli T, Fredriksen K, Osbakk SA, Bartnes K. Evaluation of a university hospital trauma team activation protocol. Scand $\mathrm{J}$ Trauma Resusc Emerg Med. 2011;19:18.

7. Sasser S, Varghese M, Kellermann A, Lormand J. Prehospital trauma care systems. Geneva: World Health Organization; 2005.

8. ACS-COT. Resources for optimal care of the injured patient. American College of Surgeons; 2006.

9. Nordgarden T, Odland P, Guttormsen AB, Ugelvik KS. Undertriage of major trauma patients at a university hospital: a retrospective cohort study. Scand J Trauma Resusc Emerg Med. 2018;26(1):64.

10. Uleberg O, Vinjevoll OP, Eriksson U, Aadahl P, Skogvoll E. Overtriage in trauma-what are the causes? Acta Anaesthesiol Scand. 2007;51(9):1178-83.

11. von Elm E, Altman DG, Egger M, Pocock SJ, Gotzsche PC, Vandenbroucke JP, Initiative S. The Strengthening the Reporting of Observational Studies in Epidemiology (STROBE) statement: guidelines for reporting observational studies. Lancet. 2007;370(9596):1453-7.

12. Rehn M, Lossius HM, Tjosevik KE, Vetrhus M, Ostebo O, Eken $\mathrm{T}$. Efficacy of a two-tiered trauma team activation protocol in a Norwegian trauma centre. Br J Surg. 2012;99(2):199-208.

13. Baker SP, O'Neill B, Haddon W Jr, Long WB. The injury severity score: a method for describing patients with multiple injuries and evaluating emergency care. J Trauma. 1974;14(3):187-96.

14. Association for the Advancement of Automotive Medicine. AIS: the Abbreviated Injury Scale (c) 1990 revision-Update-98. In Des Plains I-U.

15. van Rein EAJ, van der Sluijs R, Voskens FJ, Lansink KWW, Houwert RM, Lichtveld RA, de Jongh MA, Dijkgraaf MGW, Champion HR, Beeres FJP, et al. Development and validation of a prediction model for prehospital triage of trauma patients. JAMA Surg. 2019;154(5):421-9.

16. Stordahl H, Passas E, Hopland A, Nielsen EW. Nine out of ten trauma calls to a Norwegian hospital are avoidable: a retrospective analysis. BMC Emerg Med. 2015;15:1.

17. Fernandez FB, Ong A, Martin AP, Schwab CW, Wasser T, Butts CA, McNicholas AR, Muller AL, Barbera CF, Trupp R, et al. Success of an expedited emergency department triage evaluation system for geriatric trauma patients not meeting trauma activation criteria. Open Access Emerg Med. 2019;11:241-7. 